Research Article

\title{
Thermodynamical Study of FRW Universe in Quasi-Topological Theory
}

\author{
H. Moradpour and R. Dehghani \\ Research Institute for Astronomy and Astrophysics of Maragha (RIAAM), P.O. Box 55134-441, Maragha, Iran \\ Correspondence should be addressed to H. Moradpour; hn.moradpour@gmail.com
}

Received 8 March 2016; Accepted 19 April 2016

Academic Editor: Ahmed Farag Ali

Copyright ( $) 2016$ H. Moradpour and R. Dehghani. This is an open access article distributed under the Creative Commons Attribution License, which permits unrestricted use, distribution, and reproduction in any medium, provided the original work is properly cited. The publication of this article was funded by SCOAP ${ }^{3}$.

\begin{abstract}
By applying the unified first law of thermodynamics on the apparent horizon of FRW universe, we get the entropy relation for the apparent horizon in quasi-topological gravity theory. Throughout the paper, the results of considering the Hayward-Kodama and Cai-Kim temperatures are also addressed. Our study shows that whenever there is no energy exchange between the various parts of cosmos, we can get an expression for the apparent horizon entropy in quasi-topological gravity, which is in agreement with other attempts that followed different approaches. The effects of a mutual interaction between the various parts of cosmos on the apparent horizon entropy as well as the validity of second law of thermodynamics in quasi-topological gravity are perused.
\end{abstract}

\section{Introduction}

Since observational data indicates an accelerating universe [1-4], one should either consider a nontrivial fluid in the Einstein relativity [5-7] or modifying the Einstein theory [7-10]. Moreover, there are some observational evidences which permit a mutual interaction between the dark sides of cosmos [11-25], including the dark energy and dark matter [26]. The thermodynamic consequences of such interactions in the Einstein framework are addressed in [27]. Recently, it is argued that since the origin of dark energy is unknown, some dark energy candidates may affect the Bekenstein entropy of apparent horizon in a flat FRW universe [28-31]. In addition, by following [32], one can see that all of the dark energy candidates and their interaction with other parts of cosmos may affect the apparent horizon of a flat FRW universe in the Einstein framework. There are additional terms to the Einstein tensor in modified theories of gravity. Since these additional terms can be interpreted as a geometrical fluid, a mutual interaction between the dark sides of cosmos may be an interaction between these geometrical and material terms [10]. Therefore, one may expect that such modifications to Einstein theory and their interaction with other parts of cosmos may also affect the horizon entropy. Indeed, there are some new attempts in which authors give a positive answer to this expectation and show that such geometrical fluids and their interaction with other parts of cosmos may also affect the horizon entropy of FRW universe [33-36].

String theory together with AdS/CFT correspondence conjecture gives us the motivation to study space-times with dimensions more than 4 [37-39]. Moreover, brane scenarios also encourage us to study $(4+1)$-dimensional space-times [40, 41]. The backbone of Einstein-Hilbert action is producing the second-order field equations of motion. In looking for a suitable Lagrangian for higher dimensions, which also keeps the field equations of motion for metric of second-order, one reaches to Lovelock Lagrangian instead of the usual $(n+1)$ version of Einstein-Hilbert action [42]. It is shown that the corresponding gravitational field equations of static spherically symmetric space-times in the Einstein and Lovelock theories are parallel to the availability of the first law of thermodynamics on the horizons of considered metric [43]. For a FRW universe, where the field equations govern the universe expansion history, one can also reach the corresponding Friedmann equations in the Einstein and Lovelock theories by applying the first law of thermodynamics on the apparent horizon of FRW universe [44]. Indeed, such generalization of Einstein-Hilbert action leads to additional terms besides the Einstein tensor in spacetimes with dimensions more than 4. Authors in [33] interpret 
such additional terms as the geometrical fluids and show that these terms and their interactions with other parts of cosmos affect the horizon entropy.

By considering cubic and higher curvature interactions, some authors try to find new Lagrangian which keeps the field equation of motion for the metric of second-order [4547]. Their theory is now called quasi-topological gravity which affects the gravitational field equations in space-time with dimensions more than 4 . Thermodynamics of static black holes are studied in this theory which leads to an expression for the horizon entropy [45-50]. Moreover, it is shown that if one assumes that the black hole entropy relation, derived in [46-50], is available for the apparent horizon of FRW universe, then, by applying the unified first law of thermodynamics on the apparent horizon of FRW universe, one can get the corresponding Friedmann equations in quasitopological gravity [51]. Thereinafter, authors point out the second law of thermodynamics and its generalized form [51]. In fact, this scheme proposes that, in quasi-topological gravity, one may generalize the black hole entropy to the cosmological setup by inserting the apparent horizon radii instead of the event horizon radii. Is it the only entropy relation for the apparent horizon? Does a mutual interaction between the geometrical fluid, arising from the terms besides the Einstein tensor in the field equations, and other parts of cosmos affect the horizon entropy?

Our aim in this paper is to study the thermodynamics of apparent horizon of FRW universe in the quasi-topological gravity theory to provide proper answers for the abovementioned questions. In this investigation, we point to the results of considering the Hayward-Kodama and Cai-Kim temperatures for the apparent horizon. In order to achieve this goal, by looking at the higher-order curvature terms, arising in quasi-topological gravity, as a geometrical fluid and applying the unified first law of thermodynamics on the apparent horizon, we get an expression for the apparent horizon entropy in this theory, while the geometrical fluid does not interact with other parts of cosmos. Moreover, we focus on a FRW universe in which the geometrical fluid interacts with other parts of cosmos. This study signals us to the effects of such mutual interaction on the apparent horizon entropy of a FRW universe with arbitrary curvature for quasi-topological gravity theory. As the noninteracting case, the second law of thermodynamics is also studied in an interacting universe.

The paper is organized as follows. In the next section, we give an introductory note about the quasi-topological gravity, the corresponding Friedmann equations, and some properties of FRW universe, including its apparent horizon radii, surface gravity, and thus the corresponding HaywardKodama temperature. Section 3 is devoted to a brief discussion about the Unified First Law (UFL) of thermodynamics. In Section 4, bearing the Hayward-Kodama temperature together with the unified first law of thermodynamics in mind, we study the effect of a geometrical (curvature) fluid on the apparent horizon entropy in a FRW universe with arbitrary curvature parameter for quasi-topological theory. The results of considering the Cai-Kim temperature are also addressed. Thereinafter, we generalize our study to a universe in which the geometrical fluid interacts with other parts of cosmos. The results of attributing the Cai-Kim temperature to the apparent horizon in an interacting universe are also pointed out in Section 4. Section 5 includes some notes about the availability of second law of thermodynamics in interacting cosmos described by quasi-topological gravity theory. The final section is devoted to summary and concluding remarks.

\section{A Brief Overview of Quasi-Topological Gravity}

A natural generalization of the Einstein-Hilbert action to higher dimensional space-time, and higher-order gravity with second-order equation of motion, is the Lovelock action

$$
I_{G}=\frac{1}{16 \pi G_{n+1}} \int d^{n+1} x \sqrt{-g}\left(\sum_{i=1}^{m} c_{i} \mathscr{L}_{i}+\mathscr{L}_{M}\right),
$$

where $c_{i}$ 's are Lovelock coefficients, $\mathscr{L}_{i}$ 's are dimensionally extended Euler densities, and $\mathscr{L}_{M}$ is the matter Lagrangian. In the above action because of the topological origin of the Lovelock terms, the term proportional to $c_{i}$ contributes to the equations of motion in dimensions with $n \geq 2 m$, where $m$ is the order of Lovelock theory. Generally, although the equations of motion of $k$ th-order Lovelock gravity are second-order differential equations, the $k$ th-order Lovelock term has no contribution to the field equations in $2 k$ and lower dimensions. For example, the cubic term $\mathscr{L}_{3}$ does not have any dynamical effect in five dimensions. Recently, a modification of Lovelock gravity called quasi-topological gravity has been introduced, which has contribution to the field equations in five dimensions from the $m$ th-order $(m=$ 3) term in Riemann tensor. Several aspects of $m$ th-order quasi-topological terms which have at most second-order derivatives of the metric in the field equations for spherically symmetric space-times in five and higher dimensions except $2 p$ dimensions have been investigated.

The action of 4 th-order quasi-topological gravity in $(n+1)$ dimensions can be written as follows:

$$
\begin{aligned}
I_{G} & =\frac{1}{16 \pi G_{n+1}} \int d^{n+1} x \sqrt{-g}\left[\mu_{1} \mathscr{L}_{1}+\mu_{2} \mathscr{L}_{2}+\mu_{3} \mathscr{X}_{3}\right. \\
& \left.+\mu_{4} \mathscr{X}_{4}+\mathscr{L}_{\text {matter }}\right],
\end{aligned}
$$

which not only works in five dimensions but also yields second-order equations of motion for spherically symmetric space-times.

In action (2), $\mathscr{L}_{1}=R$ is just the Einstein-Hilbert Lagrangian, $\mathscr{L}_{2}=R_{a b c d} R^{a b c d}-4 R_{a b} R^{a b}+R^{2}$ is the secondorder Lovelock (Gauss-Bonnet) Lagrangian, and $\mathscr{L}_{\text {matter }}$ is the Lagrangian of the matter field. $\mathscr{X}_{3}$ is the curvature-cubed Lagrangian given by [46]

$$
\begin{aligned}
\mathscr{X}_{3} & =R_{a b}^{c d} R_{c d}^{e f} R_{e f}^{a b} \\
& +\frac{1}{(2 n-1)(n-3)}\left(\frac{3(3 n-5)}{8} R_{a b c d} R^{a b c d} R\right. \\
& -3(n-1) R_{a b c d} R_{e}^{a b c} R^{d e}+3(n+1) R_{a b c d} R^{a c} R^{b d}
\end{aligned}
$$




$$
\begin{aligned}
& +6(n-1) R_{a}^{b} R_{b}^{c} R_{c}^{a}-\frac{3(3 n-1)}{2} R_{a}^{b} R_{b}^{a} R \\
& \left.+\frac{3(n+1)}{8} R^{3}\right)
\end{aligned}
$$

and $\mathscr{X}_{4}$ is the fourth-order term of quasi-topological gravity [47]:

$$
\begin{aligned}
\mathscr{X}_{4}= & c_{1} R_{a b c d} R^{c d e f} R_{e f}^{h g} R_{h g}^{a b}+c_{2} R_{a b c d} R^{a b c d} R_{e f} R^{e f} \\
& +c_{3} R R_{a b} R^{a c} R_{c}^{b}+c_{4}\left(R_{a b c d} R^{a b c d}\right)^{2} \\
& +c_{5} R_{a b} R^{a c} R_{c d} R^{d b}+c_{6} R_{a b c d} R^{a c} R^{d b} \\
& +c_{7} R_{a b c d} R^{a c} R^{b e} R_{e}^{d}+c_{8} R_{a b c d} R^{a c e f} R_{e}^{b} R_{f}^{d} \\
& +c_{9} R_{a b c d} R^{a c} R_{e f} R^{b e d f}+c_{10} R^{4}+c_{11} R^{2} R_{a b c d} R^{a b c d} \\
& +c_{12} R^{2} R_{a b} R^{a b}+c_{13} R_{a b c d} R^{a b e f} R_{e f g}^{c} R^{d g} \\
& +c_{14} R_{a b c d} R^{a e c f} R_{g e h f} R^{g b h d} .
\end{aligned}
$$

The coefficients $c_{i}$ in the above term are given by

$$
\begin{aligned}
c_{1}= & -(n-1)\left(n^{7}-3 n^{6}-29 n^{5}+170 n^{4}-349 n^{3}\right. \\
& \left.+348 n^{2}-180 n+36\right), \\
c_{2}= & -4(n-3)\left(2 n^{6}-20 n^{5}+65 n^{4}-81 n^{3}+13 n^{2}\right. \\
& +45 n-18), \\
c_{3}= & -64(n-1)\left(3 n^{2}-8 n+3\right)\left(n^{2}-3 n+3\right), \\
c_{4}= & -\left(n^{8}-6 n^{7}+12 n^{6}-22 n^{5}+114 n^{4}-345 n^{3}\right. \\
& \left.+468 n^{2}-270 n+54\right), \\
c_{5} & =16(n-1)\left(10 n^{4}-51 n^{3}+93 n^{2}-72 n+18\right), \\
c_{6}= & -32(n-1)^{2}(n-3)^{2}\left(3 n^{2}-8 n+3\right),
\end{aligned}
$$

$$
\begin{aligned}
c_{7} & =64(n-2)(n-1)^{2}\left(4 n^{3}-18 n^{2}+27 n-9\right), \\
c_{8} & =-96(n-1)(n-2)\left(2 n^{4}-7 n^{3}+4 n^{2}+6 n-3\right), \\
c_{9} & =16(n-1)^{3}\left(2 n^{4}-26 n^{3}+93 n^{2}-117 n+36\right), \\
c_{10} & =n^{5}-31 n^{4}+168 n^{3}-360 n^{2}+330 n-90, \\
c_{11} & =2\left(6 n^{6}-67 n^{5}+311 n^{4}-742 n^{3}+936 n^{2}-576 n\right. \\
& +126), \\
c_{12} & =8\left(7 n^{5}-47 n^{4}+121 n^{3}-141 n^{2}+63 n-9\right), \\
c_{13} & =16 n(n-1)(n-2)(n-3)\left(3 n^{2}-8 n+3\right), \\
c_{14} & =8(n-1)\left(n^{7}-4 n^{6}-15 n^{5}+122 n^{4}-287 n^{3}\right. \\
& \left.+297 n^{2}-126 n+18\right) .
\end{aligned}
$$

In the context of universal thermodynamics, our universe should be a nonstationary gravitational system while, from the cosmological point of view, it should be homogeneous and isotropic. Therefore, the natural choice is the FRW universe, a dynamical spherically symmetric space-time, having only inner trapping horizon (the apparent horizon), which is described by the line element

$$
d s^{2}=h_{a b} d x^{a} d x^{b}+\tilde{r}^{2} d \Omega^{2},
$$

where $x^{0}=t, x^{1}=r, \widetilde{r}=a(t) r, a(t)$ is the scale factor of the universe with the curvature parameter $k$ with values $-1,0,1$ corresponding to the open, flat, and closed universes respectively, $h_{a b}=\operatorname{diag}\left(-1, a(t)^{2} /\left(1-k r^{2}\right)\right)$, and $d \Omega^{2}$ is the metric of $(n-1)$-dimensional unit sphere.

Varying the action (2) with respect to metric leads to [51]

$$
\sum_{i=1}^{m} \widehat{\mu}_{i} l^{2 i-2}\left(H^{2}+\frac{k}{a^{2}}\right)^{i}=\frac{16 \pi G_{n+1}}{n(n-1)} \rho .
$$

This is the Friedmann equation of arbitrary-order quasitopological cosmology where $H$ is the Hubble parameter. From now on, we set $G_{n+1}=1$ for simplicity. Moreover, $\widehat{\mu}_{i}$ 's are dimensionless parameters as follows:

$$
\begin{aligned}
& \widehat{\mu}_{1}=1, \\
& \widehat{\mu}_{2}=\frac{(n-2)(n-3)}{l^{2}} \mu_{2}, \\
& \widehat{\mu}_{3}=\frac{(n-2)(n-5)\left(3 n^{2}-9 n+4\right)}{8(2 n-1) l^{4}} \mu_{3}, \\
& \widehat{\mu}_{4}=\frac{n(n-1)(n-2)^{2}(n-3)(n-7)\left(n^{5}-15 n^{4}+72 n^{3}-156 n^{2}+150 n-42\right)}{l^{6}} \mu_{4} .
\end{aligned}
$$


The dynamical apparent horizon is determined by the relation $h^{a b} \partial_{a} \widetilde{r} \partial_{b} \widetilde{r}=0$. It is a matter of calculation to show that the radius of the apparent horizon for the FRW universe is [52]

$$
\tilde{r}_{A}=\frac{1}{\sqrt{H^{2}+k / a^{2}}} .
$$

In cosmological context, various definitions of temperature are used to get the corresponding Friedmann equations on the apparent horizon. First, we use the original definition of temperature (Hayward-Kodama temperature) together with the Clausius relation $\left(T d S_{A}=d Q^{m}\right)$ as well as the unified form of the first law of thermodynamics to extract an expression for the entropy of apparent horizon in the quasitopological cosmology. The Hayward-Kodama temperature associated with the apparent horizon is defined as $T_{h}=\kappa / 2 \pi$, where $\kappa$ is the surface gravity which can be evaluated by using $\kappa=(1 / 2 \sqrt{-h}) \partial_{a}\left(\sqrt{-h} h^{a b} \partial_{b} \widetilde{r}\right)[53-58]$. Therefore, the surface gravity at the apparent horizon of the FRW universe has the following form:

$$
\kappa=-\frac{1}{\widetilde{r}_{A}}\left(1-\frac{\dot{\tilde{r}}_{A}}{2 H \widetilde{r}_{A}}\right),
$$

which leads to

$$
T_{h}=\frac{\kappa}{2 \pi}=-\frac{1}{2 \pi \widetilde{r}_{A}}\left(1-\frac{\dot{\tilde{r}}_{A}}{2 H \widetilde{r}_{A}}\right),
$$

for the Hayward-Kodama temperature of apparent horizon. Then, using the Cai-Kim temperature and the Clausius relation, we get the entropy of apparent horizon. In this approach, the horizon temperature is $[44,59]$

$$
T=\frac{1}{2 \pi \widetilde{r}_{A}} .
$$

In obtaining the above relation, we consider an infinitesimal time interval, so the horizon radius will have a small change and we can use the $d \widetilde{r}_{A}=0$ approximation $[44,59]$.

\section{Unified First Law of Thermodynamics and Horizon Entropy}

The unified first law of thermodynamics can be expressed as $[52,60,61]$

$$
d E=A \Psi+W d V
$$

where $E$ is the total baryonic energy content of the universe inside an $n$-sphere of volume $V$, while $A$ is the area of the horizon. The energy flux $\Psi$ is termed as the energy supply vector and $W$ is the work function which are, respectively, defined as

$$
\begin{aligned}
A \Psi & =A\left(T_{a}^{b} \partial_{b} \widetilde{r}_{A}+W \partial_{a} \widetilde{r}_{A}\right), \\
W & =-\frac{1}{2} T^{a b} h_{a b} .
\end{aligned}
$$

The term $W d V$ in the first law comes from the fact that we have a volume change for the total system enveloped by the apparent horizon. For a pure de Sitter space, $\rho=p$, and the work term reduces to the standard $p d V$; thus we obtain exactly the standard first law of thermodynamics, $d E=T d S-$ $p d V$. It is a matter of calculation to show that (14) can be written as

$$
A \Psi=-A H \widetilde{r}_{A}\left(\frac{\rho+p}{2}\right) d t+A a\left(\frac{\rho+p}{2}\right) d r_{A}
$$

and thus

$$
\begin{aligned}
A \Psi= & -\frac{3 V(\rho+p) H}{2} d t \\
& +\frac{A(\rho+p)}{2}\left(d \widetilde{r}_{A}-\widetilde{r}_{A} H d t\right),
\end{aligned}
$$

on the apparent horizon of FRW universe. In obtaining the last equation, we used $A \widetilde{r}_{A}=3 V$ relation.

\section{Thermodynamics of Apparent Horizon in Quasi-Topological Gravity Theory}

Our universe is undergoing an accelerating expansion which represents a new imbalance in the governing Friedmann equations. Physicists have addressed such imbalances either by introducing new sources or by changing the governing equations. The standard cosmology model addresses this imbalance by introducing a new source (dark energy) in the Friedmann equations. On the contrary, a group of physicists have explored the second route, that is, a modified gravity approach, that, at large scales, Einstein theory of general relativity breaks down and a more general action describes the gravitational field. In this study, we follow the second approach.

The Friedmann equation of FRW universe in quasitopological gravity is represented by (7). In modified gravity theories, the modified Friedmann equations can be written as

$$
\begin{aligned}
H^{2}+\frac{k}{a^{2}} & =\frac{16 \pi}{n(n-1)} \rho_{t}, \\
\dot{H}-\frac{k}{a^{2}} & =-\frac{8 \pi}{(n-1)}\left(\rho_{t}+p_{t}\right) .
\end{aligned}
$$

In the above equations $\rho_{t}$ is the total energy density which includes two noninteracting fluid systems: one is the usual fluid of energy density $\rho$ and thermodynamic pressure $p$, while the second one is termed as effective energy density $\rho_{e}$ due to curvature contributions and its corresponding pressure $p_{e}$. So, we have

$$
\begin{aligned}
\rho_{t} & =\rho+\rho_{e}, \\
\rho_{t}+p_{t} & =(\rho+p)+\left(\rho_{e}+p_{e}\right),
\end{aligned}
$$


where

$$
\begin{array}{r}
\rho_{e}=-\frac{n(n-1)}{16 \pi} \sum_{i=2}^{m} \frac{\widehat{\mu}_{i} l^{2 i-2}}{\widetilde{r}_{A}^{2 i}}, \\
\rho_{e}+p_{e}=-\frac{\varepsilon(n-1)}{4 \pi} \sum_{i=2}^{m} \frac{i \widehat{\mu}_{i} l^{2 i-2}}{\widetilde{r}_{A}^{2 i}},
\end{array}
$$

where we have defined $\varepsilon=\dot{\vec{r}}_{A} / 2 H \widetilde{r}_{A}$, in which $\dot{\vec{r}}_{A}=d \widetilde{r}_{A} / d t$, and, in terms of the horizon radius $\widetilde{r}_{A}$, we have [54]

$$
\dot{H}-\frac{k}{a^{2}}=-\frac{2 \varepsilon}{\widetilde{r}_{A}^{2}} .
$$

Finally, we should note that, by combining (21) and (22), we get $\dot{\rho}_{e}+n H\left(\rho_{e}+p_{e}\right)=0$. It means that (21) and (22) are only valid if the $\dot{\rho}_{e}+n H\left(\rho_{e}+p_{e}\right)=0$ and $\dot{\rho}+n H(\rho+$ $p)=0$ conditions are simultaneously available. These results demonstrate that there is no energy exchange between the geometrical and material fluids.

4.1. Noninteracting Case. The terms due to the curvature formally play the role of a further source term in the field equations whose effect is the same as that of an effective fluid of purely geometrical origin. Indeed, since such terms modify the Einstein theory, they lead to changing the Bekenstein relation for the entropy of black hole horizon [46-49], which is in agreement with the result obtained by applying the first law of thermodynamics on the black hole horizon [50]. In order to study the thermodynamics of apparent horizon of FRW universe in quasi-topological gravity, authors in [51] assumed that the black hole entropy expression, derived in [46-50], is also valid for the apparent horizon of FRW universe. In addition, by applying the UFL of thermodynamics on the apparent horizon, they could get the Friedmann equation in the quasi-topological gravity [51]. In fact, their recipe is a way of proposing an expression for the apparent horizon entropy instead of a way for deriving the corresponding entropy. Therefore, the inverse of their recipe may indeed be considered theoretically as a more acceptable way of getting the apparent horizon entropy. Finally, based on approach [51], it seems that their proposal for the apparent horizon entropy is independent of any interaction between the cosmos sectors. It is also worthy to note that the mutual interaction between the geometrical $\left(T_{\mu \nu}^{e}\right)$ and nongeometrical $\left(T_{\mu \nu}^{m}\right)$ sectors of cosmos in various modified theories of gravity affects the apparent horizon entropy [33-36]. In fact, depending on the scales, it is such a curvature fluid which may play the role of dark energy. In Einstein general relativity framework, a dark energy candidate and its interaction with other parts of cosmos affect the Bekenstein entropy [28-32]. Therefore, the results obtained in [33-36] are in line with those of [2832]. In order to get the effects of mutual interaction between the geometrical and nongeometrical parts of cosmos, we need to establish a recipe to find out the horizon entropy by starting from the Friedmann equations and applying the UFL of thermodynamics on the apparent horizon of FRW universe. The energy conservation law leads to the continuity equation in the form

$$
\dot{\rho}_{t}+n H\left(\rho_{t}+p_{t}\right)=0 .
$$

For a noninteracting system it breaks down to

$$
\begin{aligned}
\dot{\rho}+n H(\rho+p) & =0, \\
\dot{\rho}_{e}+n H\left(\rho_{e}+p_{e}\right) & =0,
\end{aligned}
$$

which implies that (21) and (22) are available. Differentiating (18), we reach

$$
-\frac{2}{\widetilde{r}_{A}^{3}} d \widetilde{r}_{A}-\frac{16 \pi}{n(n-1)} d \rho_{e}=\frac{16 \pi}{n(n-1)} d \rho .
$$

Bearing (25) in mind, we have

$$
\frac{d \widetilde{r}_{A}}{\widetilde{r}_{A}^{3}}+\frac{8 \pi}{n(n-1)} d \rho_{e}=\frac{8 \pi}{n-1} H(\rho+p) d t .
$$

Multiplying both sides of the above equation by $(-T)$, one obtains

$$
\begin{aligned}
& (-T)\left(\frac{d \widetilde{r}_{A}}{\widetilde{r}_{A}^{3}}+\frac{8 \pi}{n(n-1)} d \rho_{e}\right) \\
& \quad=\frac{8 \pi}{n-1} H(\rho+p) d t\left[\frac{1}{2 \pi \widetilde{r}_{A}}\left(1-\frac{\dot{\tilde{r}}_{A}}{2 H \widetilde{r}_{A}}\right)\right] .
\end{aligned}
$$

Assume that the total energy content of the universe inside an $n$-sphere of radius $\widetilde{r}_{A}$ is $E=\rho V$, where $V=\Omega_{n} \widetilde{r}_{A}^{n}$ is the volume enveloped by an $n$-dimensional sphere. Taking differential form of the total energy, after using the continuity equation (25), we obtain

$$
d E=n \Omega_{n} \rho \widetilde{r}_{A}^{n-1} d \widetilde{r}_{A}-n H \Omega_{n} \widetilde{r}_{A}^{n}(\rho+p) d t,
$$

which leads to

$$
(\rho+p) d t=-\frac{d E}{n H \Omega_{n} \widetilde{r}_{A}^{n}}+\frac{\rho d \widetilde{r}_{A}}{H \widetilde{r}_{A}} .
$$

Substituting (30) into (28), simple calculations lead to

$$
\begin{aligned}
& T\left(\frac{d \widetilde{r}_{A}}{\widetilde{r}_{A}^{3}}+\frac{8 \pi}{n(n-1)} d \rho_{e}\right) \\
& \quad=\frac{4 d E}{n(n-1) \Omega_{n} \widetilde{r}_{A}^{n+1}}-\frac{2(\rho-p) d \widetilde{r}_{A}}{(n-1) \widetilde{r}_{A}^{2}},
\end{aligned}
$$

and consequently

$$
\begin{aligned}
& T\left(\frac{d \widetilde{r}_{A}}{\widetilde{r}_{A}^{3}}+\frac{8 \pi}{n(n-1)} d \rho_{e}\right) \\
& \quad=\frac{4}{n(n-1) \Omega_{n} \widetilde{r}_{A}^{n+1}}[d E-W d V],
\end{aligned}
$$

where we have used $d V=n \Omega_{n} \widetilde{r}_{A}^{n-1} d \widetilde{r}_{A}$. In this equation the work density $W=(\rho-p) / 2$ is regarded as the work done 
when the apparent horizon radius changes from $\widetilde{r}_{A}$ to $\widetilde{r}_{A}+$ $d \widetilde{r}_{A}$. The Clausius relation is [32]

$$
T d S_{A}=\delta Q^{m}=A \Psi,
$$

where $\delta Q^{m}$ is the energy flux crossing the horizon during the universe expansion. Considering (13), (32), and (33), we have

$$
d S_{A}=\frac{n(n-1) \Omega_{n} \widetilde{r}_{A}^{n-2} d \widetilde{r}_{A}}{4}+2 \pi \Omega_{n} \widetilde{r}_{A}^{n+1} d \rho_{e} .
$$

Using (25) leads to

$$
\begin{aligned}
d S_{A}= & \frac{n(n-1) \Omega_{n} \widetilde{r}_{A}^{n-2} d \widetilde{r}_{A}}{4} \\
& -2 n H \pi \Omega_{n} \widetilde{r}_{A}^{n+1}\left(\rho_{e}+p_{e}\right) d t .
\end{aligned}
$$

Now, by either inserting (21) into (34) or (22) into (35) and integrating the result, we get

$$
S_{A}=\frac{A}{4} \sum_{i=1}^{m} i \frac{(n-1)}{(n-2 i+1)} \frac{\widehat{\mu}_{i} l^{2 i-2}}{\widetilde{r}_{A}^{2 i-2}}+S_{0},
$$

for the horizon entropy. This result is in full agreement with previous proposal in which authors assumed that the event horizon entropy is extendable to the apparent horizon of FRW universe [51]. $S_{0}$ is an integration constant and we can consider it zero without loss of generality. It is also useful to mention here that we considered the Clausius relation in the form $T d S_{A}=\delta Q^{m}$ to obtain this result, while authors in [51] took into account the $T d S_{A}=-\delta Q^{m}$ form of the Clausius relation. This discrepancy is due to our different way of defining the horizon temperature. We have used the Hayward-Kodama definition of temperature (11), while authors in [51] used its absolute value to avoid attributing negative temperatures on the apparent horizon and thus the Hawking radiation.

The Cai-Kim Approach. Here, we focus on the Cai-Kim approach to get the effects of geometrical fluid on the horizon entropy. When one applies the first law on the apparent horizon to calculate the surface gravity and thereby the temperature and considers an infinitesimal amount of energy crossing the apparent horizon, the apparent horizon radius $\tilde{r}_{A}$ should be regarded to have a fixed value $(d V=0)$ [59]. Bearing (17) together with $d \widetilde{r}_{A}=0$ approximation in mind, by using (25) and the Clausius relation in the form $T d S_{A}=$ $-\delta Q^{m}=-A \Psi$, where $\delta Q^{m}$ is the energy flux crossing the horizon during the infinitesimal time interval $d t$, we get

$$
T d S_{A}=-V d \rho .
$$

Substituting $d \rho$ from (26) into the above equation, we are led to

$$
d S_{A}=-\frac{V}{T} d \rho=2 \pi \Omega_{n} \widetilde{r}_{A}^{n+1}\left[\frac{n(n-1) d \widetilde{r}_{A}}{8 \pi \widetilde{r}_{A}^{3}}+d \rho_{e}\right],
$$

where we have used $V=\Omega_{n} \widetilde{r}_{A}^{n}$ and the Cai-Kim temperature $\left(T=1 / 2 \pi \tilde{r}_{A}\right)[44,59]$. Finally, we obtain

$$
d S_{A}=\frac{n(n-1) \Omega_{n} \widetilde{r}_{A}^{n-2} d \widetilde{r}_{A}}{4}+2 \pi \Omega_{n} \widetilde{r}_{A}^{n+1} d \rho_{e} .
$$

Consequently the modified entropy on the event horizon has the explicit form

$$
S_{A}=\frac{A}{4} \sum_{i=1}^{m} i \frac{(n-1)}{(n-2 i+1)} \frac{\widehat{\mu}_{i} l^{2 i-2}}{\widetilde{r}_{A}^{2 i-2}}+S_{0},
$$

which is compatible with the results obtained by considering the Hayward-Kodama temperature (36). As before, since entropy is not an absolute quantity, without loss of generality, we can set $S_{0}$ to zero. Setting $n=3$ and $i=1$ in the above result leads to the Bekenstein-Hawking entropy formula.

4.2. Interacting Universe. In order to take the interaction into account, for the quasi-topological gravity, consider the energy-momentum conservation law in the forms

$$
\begin{gathered}
\dot{\rho}+n H(\rho+p)=Q, \\
\dot{\rho}_{e}+n H\left(\rho_{e}+p_{e}\right)=-Q,
\end{gathered}
$$

where $Q$ is the mutual interaction between different parts of the cosmos. The latter means that (21) and (22) are not simultaneously valid for an interacting universe. Using (26) and (41), we get

$$
\frac{d \widetilde{r}_{A}}{\widetilde{r}_{A}^{3}}+\frac{8 \pi}{n(n-1)}\left(d \rho_{e}+Q d t\right)=\frac{8 \pi H}{n-1}(\rho+p) d t
$$

Multiplying both sides of the above equation by $(-T)$, one obtains

$$
\begin{aligned}
& T\left(\frac{d \widetilde{r}_{A}}{\widetilde{r}_{A}^{3}}+\frac{8 \pi}{n(n-1)}\left(d \rho_{e}+Q d t\right)\right) \\
& \quad=-\frac{2 H(\rho+p)}{(n-1) \widetilde{r}_{A}} d t+\frac{2(\rho+p)}{(n-1) \widetilde{r}_{A}^{2}} a d r_{A},
\end{aligned}
$$

where we have used $\dot{\tilde{r}}_{A} d t=a d r_{A}+r_{A} H d t$. Multiplying the result by the factor $n(n-1) \Omega_{n} \widetilde{r}_{A}^{n+1} / 4$, we arrive at

$$
\begin{aligned}
& T\left(\frac{d \widetilde{r}_{A}}{\widetilde{r}_{A}^{3}}+\frac{8 \pi}{n(n-1)}\left(d \rho_{e}+Q d t\right)\right) \\
& \quad \cdot\left(\frac{n(n-1) \Omega_{n} \widetilde{r}_{A}^{n+1}}{4}\right) \\
& \quad=n \Omega_{n} \widetilde{r}_{A}^{n}\left[-\frac{\rho+p}{2} H d t+\frac{\rho+p}{2 \widetilde{r}_{A}} a d r_{A}\right] .
\end{aligned}
$$

Simple calculations lead to

$$
\begin{aligned}
T & \left(\frac{n(n-1) \Omega_{n} \widetilde{r}_{A}^{n-2} d \widetilde{r}_{A}}{4}+2 \pi \Omega_{n} \widetilde{r}_{A}^{n+1}\left(d \rho_{e}+Q d t\right)\right) \\
& =-A H \frac{\rho+p}{2} \widetilde{r}_{A} d t+A a \frac{\rho+p}{2} d r_{A} .
\end{aligned}
$$


The right-hand side of (46) is nothing but the energy flux $\left(\delta Q^{m}\right)$ crossing the apparent horizon (16). Bearing the Clausius relation (33) in mind, we get

$$
\begin{aligned}
d S_{A}= & \frac{n(n-1) \Omega_{n} \widetilde{r}_{A}^{n-2} d \widetilde{r}_{A}}{4} \\
& +2 \pi \Omega_{n} \widetilde{r}_{A}^{n+1}\left(d \rho_{e}+Q d t\right) .
\end{aligned}
$$

Now, use (42) to obtain

$$
\begin{aligned}
d S_{A}= & \frac{n(n-1) \Omega_{n} \widetilde{r}_{A}^{n-2} d \widetilde{r}_{A}}{4} \\
& -2 \pi n H \Omega_{n} \widetilde{r}_{A}^{n+1}\left(\rho_{e}+p_{e}\right) d t,
\end{aligned}
$$

which leads to

$$
S_{A}=\frac{A}{4}-2 \pi n \Omega_{n} \int H \widetilde{r}_{A}^{n+1}\left(\rho_{e}+p_{e}\right) d t+S_{0},
$$

which is in agreement with the results obtained by authors [33] for the Lovelock theory but in a different way. In fact, since in the interacting universes $\rho_{e}$ and $\rho_{e}+p_{e}$ cannot simultaneously meet (21) and (22), respectively, we can not use them to integrate with the RHS of (47) and (48) to get an expression for the horizon entropy. Now, consider a special situation in which $\rho_{e}$ satisfies (21); from (47) we get

$$
\begin{aligned}
d S_{A}= & \frac{n(n-1) \Omega_{n} \widetilde{r}_{A}^{n-2} d \widetilde{r}_{A}}{4}+2 \pi \Omega_{n} \widetilde{r}_{A}^{n+1} d \rho_{e} \\
& +2 \pi \Omega_{n} \widetilde{r}_{A}^{n+1} Q d t .
\end{aligned}
$$

In the above equation, the first two terms of the right-hand side are exactly the same as (39). In other words,

$$
\left(S_{A}\right)_{\text {interaction }}=\left(S_{A}\right)_{\text {non-interaction }}+2 \pi \Omega_{n} \int \widetilde{r}_{A}^{n+1} Q d t .
$$

It is crystal clear that the second term of RHS of this equation counts the effects of mutual interaction between the geometrical and nongeometrical fluids on the horizon entropy. Loosely speaking, the above expression for horizon entropy is no longer the usual Bekenstein entropy formula; rather there are two correction terms which are due to higherorder curvature terms, arising from the quasi-topological nature of gravity theory, along with the mutual interaction between the geometrical and nongeometrical parts. Note that, in this situation, due to (42), the $\rho_{e}+p_{e}$ term does not meet (22). As another example, consider a situation in which $\rho_{e}+p_{e}$ satisfies (22), which also means that, due to (42), $\rho_{e}$ does not obey (21). For this case, by combining (42) and (47) and integrating the result, we obtain

$$
\left(S_{A}\right)_{\text {interaction }}=\left(S_{A}\right)_{\text {non-interaction }},
$$

which means that the mutual interaction $Q$ does not affect the horizon entropy in this special case.

The Cai-Kim Approach. The rest of this section is devoted to achieving (48) by using the Cai-Kim approach [44, 59]. For an interacting universe the UFL is in the form [31]

$$
d S_{A}=-\frac{V}{T}(d \rho-Q d t) .
$$

Using (26) and (42), we have

$$
d \rho-Q d t=-\frac{n(n-1) d \widetilde{r}_{A}}{8 \pi \widetilde{r}_{A}^{3}}+n H\left(\rho_{e}+p_{e}\right) d t .
$$

Substituting it into (53) leads to

$$
\begin{aligned}
d S_{A}= & \frac{n(n-1) \Omega_{n} \widetilde{r}_{A}^{n-2} d \widetilde{r}_{A}}{4} \\
& -2 \pi n H \Omega_{n} \widetilde{r}_{A}^{n+1}\left(\rho_{e}+p_{e}\right) d t,
\end{aligned}
$$

which is in full agreement with (48).

\section{Second Law of Thermodynamics}

The time evolution of the entropy in a noninteracting universe governed by quasi-topological gravity is already considered by authors in [51]. Now, we are interested in examining the validity of the second law of thermodynamics in an interacting universe. This law states that the horizon entropy should meet the $d S_{A} / d t \geq 0$ condition [62]. For this propose, from (48), we have

$$
\frac{d S_{A}}{d t}=\frac{n(n-1) \Omega_{n} \widetilde{r}_{A}^{n-2} \dot{\vec{r}}_{A}}{4}-2 \pi n H \Omega_{n} \widetilde{r}_{A}^{n+1}\left(\rho_{e}+p_{e}\right) .
$$

However, using (42) and (43), one gets

$$
\frac{\dot{\tilde{r}}_{A}}{\tilde{r}_{A}^{3}}-\frac{8 \pi H}{n-1}\left(\rho_{e}+p_{e}\right)=\frac{8 \pi H}{n-1}(\rho+p),
$$

which leads to the Raychaudhuri equation

$$
\dot{\tilde{r}}_{A}=\frac{8 \pi H}{n-1}\left(\rho+p+\rho_{e}+p_{e}\right) \widetilde{r}_{A}^{3} .
$$

Substituting it into (56), we arrive at

$$
\frac{d S_{A}}{d t}=2 \pi n H \Omega_{n} \widetilde{r}_{A}^{n+1}(\rho+p)
$$

The above result indicates that the second law of thermodynamics $\left(d S_{A} / d t \geq 0\right)$ is valid for the apparent horizon under the condition $(\rho+p)>0$ and if we define the state parameter $\omega=p / \rho$, this law is valid whenever the state parameter obeys $\omega \geq-1$.

\section{Summary and Conclusions}

After giving a brief review of the quasi-topological gravity theory, by considering a FRW universe, we pointed out to the corresponding Friedmann equation in this modified gravity theory. In Section 2, we mentioned the apparent horizon of FRW universe as the proper causal boundary, its surface gravity, and the Hayward-Kodama temperature of this hypersurface. Motivated by recent works on the thermodynamics of horizon of FRW universe in some modified gravity theories [33-36], we considered the terms other than Einstein tensor as a fluid with energy density $\rho_{e}$ and pressure $p_{e}$. In fact, since 
these terms may play the role of dark energy and because a dark energy candidate may also affect the horizon entropy [28-32], such justification at least is not forbidden. Then, we showed that if we use either the Hayward-Kodama or the Cai-Kim temperature, the same result for the horizon entropy is obtainable ((36) and (40)) which is in agreement with previous work [50], in which authors, by using different definitions for the horizon temperature and generalizing the black hole entropy to the cosmological horizon setup, could propose the same expression as ours for the apparent horizon entropy in quasi-topological gravity. Moreover, it is useful to note that (36) is valid only if there is no energy-momentum exchange between the geometrical $\left(T_{\mu \nu}^{e}\right)$ and nongeometrical $\left(T_{\mu \nu}^{m}\right)$ fluids.

Finally, we generalized our investigation to an interacting FRW universe with arbitrary curvature parameter $(k)$ in quasi-topological gravity. We got a relation for the horizon entropy (see (49)) which showed the effects of an energymomentum exchange between the geometrical and nongeometrical fluids on the apparent horizon entropy. Thereinafter, we studied the special case in which $\rho_{e}$ meets (21) to perceive clearly the effects of such mutual interaction on the horizon entropy. We got (51) for the horizon entropy, which showed two terms besides the Bekenstein entropy due to higher-order curvature terms, arising from the quasi-topological nature of model, and mutual interaction between the geometrical and nongeometrical fluids. Our investigation also showed that whenever $\rho_{e}+p_{e}$ satisfies (22), the mutual interaction $Q$ does not affect the horizon entropy and therefore the result of noninteracting case is obtainable. We have also pointed out the validity of second law of thermodynamics in an interacting case and found out that, independent of curvature parameter $(k)$, whenever $\rho+p \geq 0$, the second law is obtainable.

Our study shows that, in quasi-topological gravity theory, a geometrical fluid with energy density $\rho_{e}$ and pressure $p_{e}$, independent of its nature, affects the apparent horizon entropy as

$$
S_{A}=\frac{A}{4}-2 \pi n \Omega_{n} \int H \widetilde{r}_{A}^{n+1}\left(\rho_{e}+p_{e}\right) d t,
$$

which is in full agreement with other studies [28-36]. Since such correction in quasi-topological gravity has geometrical nature, it is indeed inevitable. Moreover, the effects of mutual interaction between the dark energy candidate and other parts of cosmos are stored in the second term of RHS of this equation. Indeed, based on our approach, one may find the apparent horizon entropy of FRW universe with arbitrary curvature parameter $(k)$ in modified theories of gravity by interpreting the terms other than Einstein tensor in the corresponding Friedmann equation as a geometrical fluid and applying the UFL of thermodynamics on the apparent horizon. At the end, we should stress that, in the absence of a mutual interaction, the results of interacting universes in quasi-topological theory converge to those of the noninteracting case.

\section{Competing Interests}

The authors declare that they have no competing interests.

\section{Acknowledgments}

This work has been supported financially by Research Institute for Astronomy \& Astrophysics of Maragha (RIAAM).

\section{References}

[1] A. G. Riess, A. V. Filippenko, P. Challis et al., "Observational evidence from supernovae for an accelerating universe and a cosmological constant," The Astronomical Journal, vol. 116, pp. 1009-1038, 1998.

[2] S. Perlmutter, G. Aldering, G. Goldhaber et al., "Measurements of $\Omega$ and $\Lambda$ from 42 high-redshift supernovae," The Astrophysical Journal, vol. 517, no. 2, article 565, 1999.

[3] R. A. Knop, G. Aldering, R. Amanullah et al., "New constraints on $\Omega_{M}, \Omega_{\Lambda}$, and $w$ from an independent set of 11 high-redshift supernovae observed with the Hubble Space Telescope," The Astrophysical Journal, vol. 598, no. 1, article 102, 2003.

[4] P. de Bernardis, P. A. R. Ade, J. J. Bock et al., "A flat Universe from high-resolution maps of the cosmic microwave background radiation," Nature, vol. 404, pp. 955-959, 2000.

[5] S. Nojiri and S. D. Odintsov, "The new form of the equation of state for dark energy fluid and accelerating universe," Physics Letters B, vol. 639, no. 3-4, pp. 144-150, 2006.

[6] M. Li, X. D. Li, S. Wang, and Y. Wang, "Dark energy," The Universe, vol. 1, no. 4, pp. 24-45, 2013.

[7] K. Bamba, S. Capozziello, S. Nojiri, and S. D. Odintsov, "Dark energy cosmology: the equivalent description via different theoretical models and cosmography tests," Astrophysics and Space Science, vol. 342, no. 1, pp. 155-228, 2012.

[8] S. Nojiri and S. D. Odintsov, "Unified cosmic history in modified gravity: from $\mathrm{F}(\mathrm{R})$ theory to Lorentz non-invariant models," Physics Reports, vol. 505, no. 2-4, pp. 59-144, 2011.

[9] S. Capozziello and V. Faraoni, Beyond Einstein Gravity, Springer, New York, NY, USA, 2011.

[10] F. S. N. Lobo, "Beyond Einstein's general relativity," Journal of Physics: Conference Series, vol. 600, no. 1, Article ID 012006, 2015.

[11] W. Zimdahl, D. Pavón, and L. P. Chimento, "Interacting quintessence," Physics Letters, Section B: Nuclear, Elementary Particle and High-Energy Physics, vol. 521, no. 3-4, pp. 133-138, 2001.

[12] L. P. Chimento, A. S. Jakubi, D. Pavón, and W. Zimdahl, "Interacting quintessence solution to the coincidence problem," Physical Review D, vol. 67, Article ID 083513, 2003.

[13] S. del Campo, R. Herrera, and D. Pavón, "Late universe expansion dominated by domain walls and dissipative dark matter," Physical Review D, vol. 70, no. 4, Article ID 043540, 2004.

[14] D. Pavón and W. Zimdahl, "Holographic dark energy and cosmic coincidence," Physics Letters, Section B: Nuclear, Elementary Particle and High-Energy Physics, vol. 628, no. 3-4, pp. 206-210, 2005.

[15] G. Olivares, F. Atrio-Barandela, and D. Pavón, "Observational constraints on interacting quintessence models," Physical Review D, vol. 71, no. 6, Article ID 063523, pp. 1-7, 2005.

[16] G. Olivares, F. Atrio-Barandela, and D. Pavón, "Matter density perturbations in interacting quintessence models," Physical Review D, vol. 74, Article ID 043521, 2006. 
[17] B. Wang, Y. Gong, and E. Abdalla, "Transition of the dark energy equation of state in an interacting holographic dark energy model," Physics Letters B, vol. 624, no. 3-4, pp. 141-146, 2005.

[18] B. Wang, Ch.-Y. Lin, and E. Abdalla, "Constraints on the interacting holographic dark energy model," Physics Letters B, vol. 637, no. 6, pp. 357-361, 2006.

[19] B. Wang, J. Zang, C.-Y. Lin, E. Abdalla, and S. Micheletti, "Interacting dark energy and dark matter: observational constraints from cosmological parameters," Nuclear Physics B, vol. 778, no. 1-2, pp. 69-84, 2007.

[20] B. Wang, C.-Y. Lin, D. Pavón, and E. Abdalla, “Thermodynamical description of the interaction between holographic dark energy and dark matter," Physics Letters B, vol. 662, no. 1, pp. $1-6,2008$.

[21] S. Das, P. S. Corasaniti, and J. Khoury, "Superacceleration as the signature of a dark sector interaction," Physical Review D, vol. 73, no. 8, Article ID 083509, 2006.

[22] L. Amendola, "Coupled quintessence," Physical Review D, vol. 62, no. 4, Article ID 043511, 2000.

[23] L. Amendola and D. Tocchini-Valentini, "Stationary dark energy: the present universe as a global attractor," Physical Review D, vol. 64, no. 4, Article ID 043509, 2001.

[24] L. Amendola and C. Quercellini, "Tracking and coupled dark energy as seen by the Wilkinson Microwave Anisotropy Probe," Physical Review D, vol. 68, no. 2, Article ID 023514, 2003.

[25] L. Amendola, S. Tsujikawa, and M. Sami, "Phantom damping of matter perturbations," Physics Letters, Section B: Nuclear, Elementary Particle and High-Energy Physics, vol. 632, no. 2-3, pp. 155-158, 2006.

[26] M. Roos, Introduction to Cosmology, John Wiley \& Sons, Chichester, UK, 2003.

[27] M. Jamil, E. N. Saridakis, and M. R. Setare, "Thermodynamics of dark energy interacting with dark matter and radiation," Physical Review D, vol. 81, no. 2, Article ID 023007, 6 pages, 2010.

[28] C.-J. Feng, X.-Z. Li, and X.-Y. Shen, "Thermodynamic of the ghost dark energy universe," Modern Physics Letters A, vol. 27, no. 31, Article ID 1250182, 11 pages, 2012.

[29] A. Sheykhi, "Entropy and thermodynamics of ghost dark energy," Canadian Journal of Physics, vol. 92, no. 6, pp. 529-532, 2014.

[30] H. Moradpour, M. T. Mohammadi Sabet, and A. Ghasemi, "Thermodynamic analysis of universes with the initial and final de Sitter eras," Modern Physics Letters A, vol. 30, no. 31, Article ID 1550158, 2015.

[31] H. Moradpour and M. T. Mohammadi Sabet, “Thermodynamic descriptions of polytropic gas and its viscous type as dark energy candidates," Canadian Journal of Physics, vol. 94, no. 3, pp. 334341, 2016.

[32] H. Ebadi and H. Moradpour, "Thermodynamics of universe with a varying dark energy component," International Journal of Modern Physics D, vol. 24, no. 14, Article ID 1550098, 2015.

[33] S. Mitra, S. Saha, and S. Chakraborty, "Universal thermodynamics in different gravity theories: conditions for generalized second law of thermodynamics and thermodynamical equilibrium on the horizons," Annals of Physics, vol. 355, pp. 1-20, 2015.

[34] S. Mitra, S. Saha, and S. Chakraborty, "A study of universal thermodynamics in Lanczos-LOVelock gravity," General Relativity and Gravitation, vol. 47, article 69, 2015.

[35] S. Mitra, S. Saha, and S. Chakraborty, "A study of universal thermodynamics in brane world scenario," Advances in High Energy Physics, vol. 2015, Article ID 430764, 9 pages, 2015.
[36] S. Saha, S. Mitra, and S. Chakraborty, "A study of universal thermodynamics in massive gravity: modified entropy on the horizons," General Relativity and Gravitation, vol. 47, no. 4, Art. 38, 17 pages, 2015.

[37] J. Polchinski, String Theory I \& II, Cambridge University Press, Cambridge, UK, 1998.

[38] J. M. Maldacena, “The large $N$ limit of superconformal field theories and supergravity," Advances in Theoretical and Mathematical Physics, vol. 2, no. 2, pp. 231-252, 1998.

[39] J. Maldacena, "The large- $N$ limit of superconformal field theories and supergravity," International Journal of Theoretical Physics, vol. 38, no. 4, p. 1113, 1999.

[40] L. Randall and R. Sundrum, "Large mass hierarchy from a small extra dimension," Physical Review Letters, vol. 83, no. 17, pp. 3370-3373, 1999.

[41] L. Randall and R. Sundrum, "An alternative to compactification,” Physical Review Letters, vol. 83, no. 23, pp. 4690-4693, 1999.

[42] D. Lovelock, "The einstein tensor and its generalizations," Journal of the Mathematical Physics, vol. 12, no. 3, p. 498, 1971.

[43] A. Paranjape, S. Sarkar, and T. Padmanabhan, "Thermodynamic route to field equations in Lanczos-LOVelock gravity," Physical Review D, vol. 74, no. 10, Article ID 104015, 9 pages, 2006.

[44] R. G. Cai and S. P. Kim, "First law of thermodynamics and Friedmann equations of Friedmann-Robertson-Walker universe," Journal of High Energy Physics, vol. 2005, no. 2, article 050, 2005.

[45] J. Oliva and S. Ray, "A new cubic theory of gravity in five dimensions: black hole, Birkhoff's theorem and C-functionfunction," Classical and Quantum Gravity, vol. 27, no. 22, Article ID 225002, 16 pages, 2010.

[46] R. C. Myers and B. Robinson, "Black holes in quasi-topological gravity," Journal of High Energy Physics, vol. 2010, article 67, 2010.

[47] M. H. Dehghani, A. Bazrafshan, R. B. Mann, M. R. Mehdizadeh, M. Ghanaatian, and M. H. Vahidinia, "Black holes in (quartic) quasitopological gravity," Physical Review D, vol. 85, no. 10, Article ID 104009, 12 pages, 2012.

[48] M. H. Dehghani and M. H. Vahidinia, "Surface terms of quasitopological gravity and thermodynamics of charged rotating black branes," Physical Review D, vol. 84, no. 8, Article ID 084044, 2011.

[49] W. G. Brenna, M. H. Dehghani, and R. B. Mann, "Quasitopological Lifshitz black holes," Physical Review D, vol. 84, no. 2, Article ID 024012, 12 pages, 2011.

[50] A. Sheykhi, M. H. Dehghani, and R. Dehghani, "Horizon thermodynamics and gravitational field equations in quasi-topological gravity," General Relativity and Gravitation, vol. 46, no. 4, pp. 1-14, 2014.

[51] M. H. Dehghani, A. Sheykhi, and R. Dehghani, "Thermodynamics of quasi-topological cosmology," Physics Letters B, vol. 724, no. 1-3, pp. 11-16, 2013.

[52] S. A. Hayward, "Unified first law of black-hole dynamics and relativistic thermodynamics," Classical and Quantum Gravity, vol. 15, no. 10, pp. 3147-3162, 1998.

[53] D. Bak and S.-J. Rey, "Cosmic holography," Classical and Quantum Gravity, vol. 17, no. 15, pp. L83-L89, 2000.

[54] R.-G. Cai and L.-M. Cao, "Unified first law and the thermodynamics of the apparent horizon in the FRW universe," Physical Review D, vol. 75, no. 6, Article ID 064008, 11 pages, 2007.

[55] R.-G. Cai and L.-M. Cao, "Thermodynamics of apparent horizon in brane world scenario," Nuclear Physics. B, vol. 785, no. 1-2, pp. 135-148, 2007. 
[56] D. W. Tian and I. Booth, "Apparent horizon and gravitational thermodynamics of the universe: solutions to the temperature and entropy confusions and extensions to modified gravity," Physical Review D, vol. 92, Article ID 024001, 2015.

[57] P. Binétruy and A. Helou, "The apparent Universe," Classical and Quantum Gravity, vol. 32, no. 20, Article ID 205006, 12 pages, 2015.

[58] A. Helou, "Dynamics of the cosmological apparent horizon: surface gravity \& temperature," http://arxiv.org/abs/1502 .04235\#.

[59] R. G. Cai, L. M. Cao, and Y. P. Hu, "Hawking radiation of an apparent horizon in a FRW universe," Classical and Quantum Gravity, vol. 26, no. 15, Article ID 155018, 2009.

[60] S. A. Hayward, "Gravitational energy in spherical symmetry," Physical Review D, vol. 53, no. 4, pp. 1938-1949, 1996.

[61] S. A. Hayward, "Energy conservation for dynamical black holes," Physical Review Letters, vol. 93, no. 25, Article ID 251101, 4 pages, 2004.

[62] S. W. Hawking, "Gravitational radiation from colliding black holes," Physical Review Letters, vol. 26, no. 21, pp. 1344-1346, 1971. 

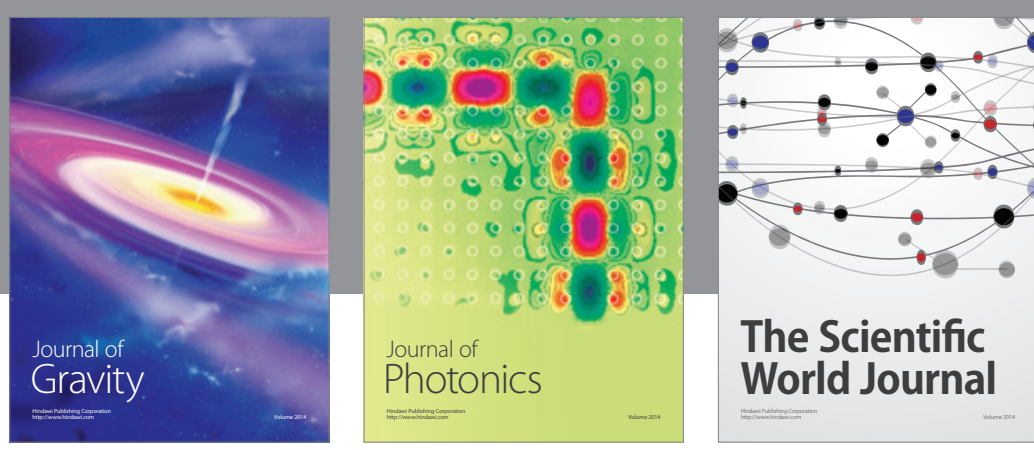

The Scientific World Journal
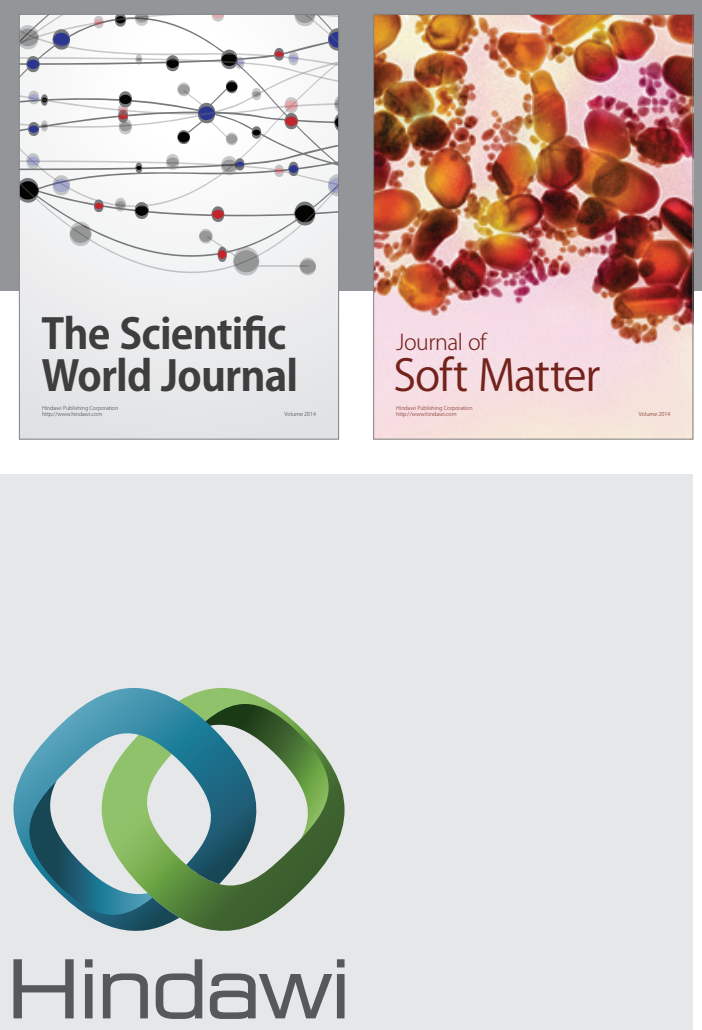

Submit your manuscripts at

http://www.hindawi.com

nternational Journal of

Statistical Mechanics
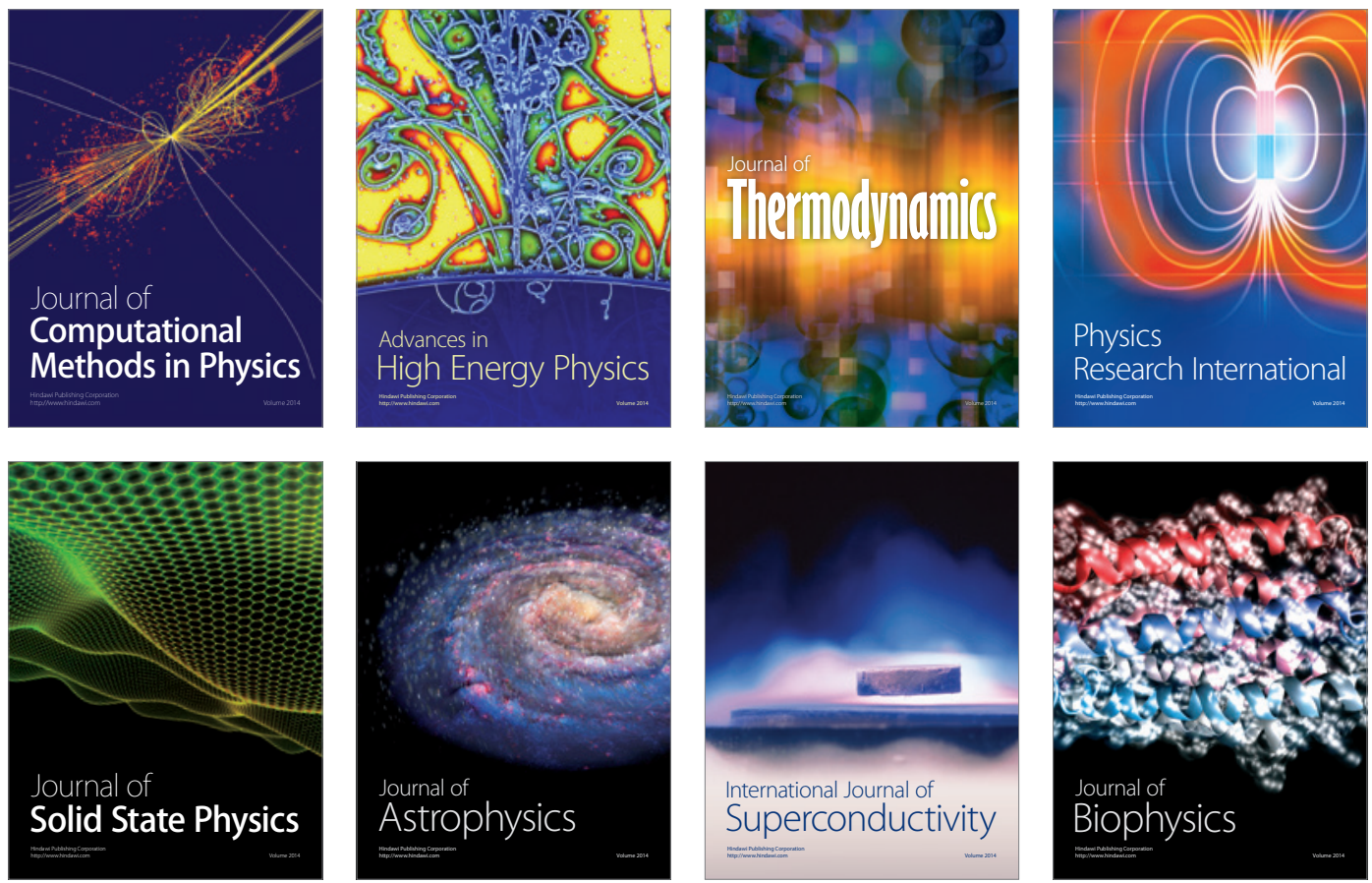
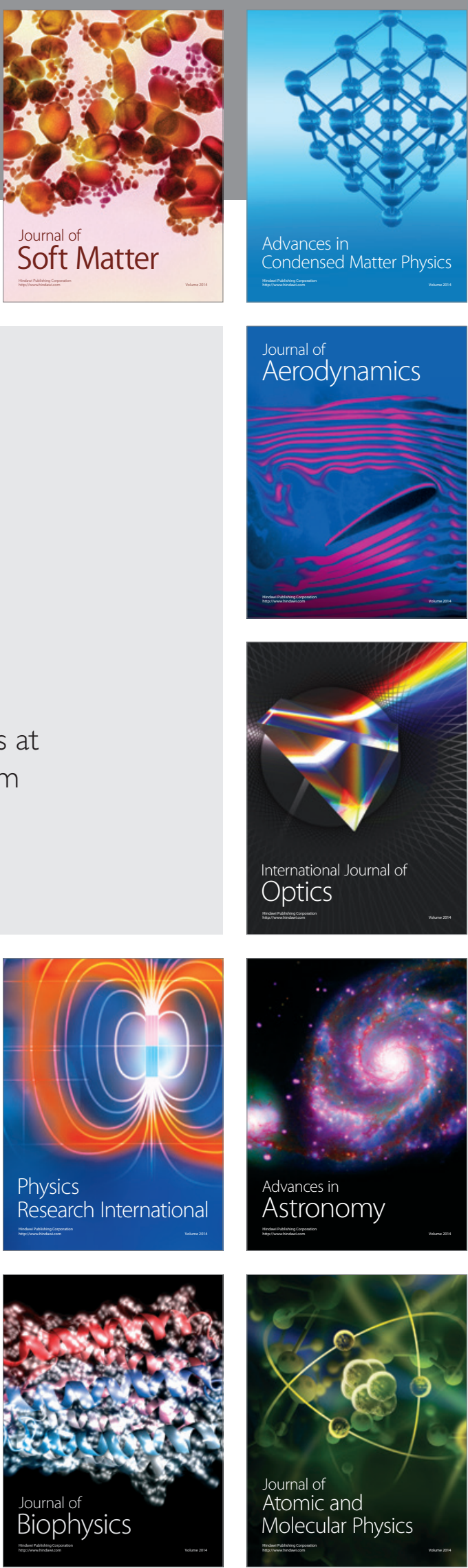